\title{
A method for merging of ultra-small-angle X-ray scattering and smeared small-angle $X$-ray scattering patterns of fibers
}

\author{
Norbert Stribeck and Ulrich Nöchel
}

J. Appl. Cryst. (2008). 41, 715-722

Copyright (C) International Union of Crystallography

Author(s) of this paper may load this reprint on their own web site or institutional repository provided that this cover page is retained. Republication of this article or its storage in electronic databases other than as specified above is not permitted without prior permission in writing from the IUCr.

For further information see http://journals.iucr.org/services/authorrights.html

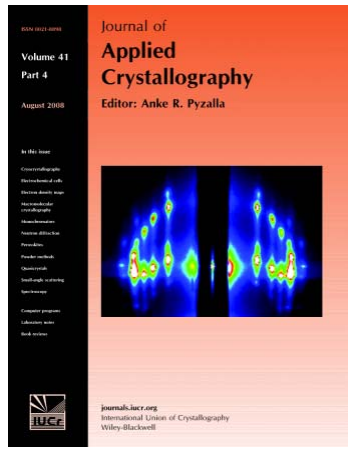

Many research topics in condensed matter research, materials science and the life sciences make use of crystallographic methods to study crystalline and non-crystalline matter with neutrons, X-rays and electrons. Articles published in the Journal of Applied Crystallography focus on these methods and their use in identifying structural and diffusioncontrolled phase transformations, structure-property relationships, structural changes of defects, interfaces and surfaces, etc. Developments of instrumentation and crystallographic apparatus, theory and interpretation, numerical analysis and other related subjects are also covered. The journal is the primary place where crystallographic computer program information is published.

\section{Crystallography Journals Online is available from journals.iucr.org}


Journal of

Applied

Crystallography

ISSN 0021-8898

Received 23 November 2007

Accepted 18 May 2008

\section{A method for merging of ultra-small-angle X-ray scattering and smeared small-angle $X$-ray scattering patterns of fibers}

\author{
Norbert Stribeck* and Ulrich Nöchel \\ Institute of Technical and Macromolecular Chemistry, University of Hamburg, Bundesstrasse 45, \\ D-20146 Hamburg, Germany. Correspondence e-mail: norbert.stribeck@desy.de
}

\begin{abstract}
A method for collimation correction and combination of anisotropic scattering patterns recorded in tandem experiments is proposed. It includes both an advanced two-dimensional extrapolation procedure for the center of the pattern and (compared with the 'blind deconvolution' method) a more justified procedure for desmearing from an unknown broad primary beam profile. This semi-blind deconvolution rests on the availability of unsmeared data in a region of the smeared image. Materials exhibiting both ultra-small-angle X-ray scattering (USAXS) and small-angle X-ray scattering (SAXS) must be studied in both angular bands (tandem experiment), in order to collect the complete range of discrete scattering for nanostructure analysis. Merging of the patterns requires desmearing of at least the SAXS pattern from its point-spread function, i.e. the primary beam profile. The distorting effect of single-band experiments on the reconstructed nanostructure of polymer materials is demonstrated.
\end{abstract}

(C) 2008 International Union of Crystallography Printed in Singapore - all rights reserved primary beam profiles in order to cover the complete angular range in which discrete scattering is observed. Frequently, the corresponding materials exhibit both SAXS and ultra-smallangle X-ray scattering (USAXS). Comparing the two patterns, the smeared nature of the SAXS pattern is readily established. Fig. 1 demonstrates that pattern merging cannot be successful if the SAXS pattern is not desmeared. As far as we know, a desmearing method for two-dimensional scattering data has not yet been published.

Finally, we define the term 'discrete scattering'. A material for which the interference function (Porod, 1967; Ruland, 1977; Stribeck, 2001, 2007) does not vanish is showing discrete scattering.

\section{Experimental and common data evaluation}

\subsection{Materials}

Different polymer materials were investigated [hard-elastic polypropylene (PP), PP nanofibers, fibers from polyethylene terephthalate/PP blends] in heat load experiments or during mechanical testing. For the purpose of structure evolution studies, the experiments were monitored by X-ray scattering both in a USAXS setup and - during a repetition of the experiment - in a SAXS setup ('tandem experiment').

\subsection{USAXS}

USAXS was performed at the synchrotron beamline BW4 at HASYLAB, Hamburg. The wavelength of the radiation was $0.13 \mathrm{~nm}$. The sample-detector distance ranged between 8 and $13 \mathrm{~m}$. Scattering patterns were collected by a two-dimensional 
position-sensitive marCCD 165 detector (Marresearch, Norderstedt, Germany) operated in $2048 \times 2048$ pixel mode (79 $\mu \mathrm{m}$ quadratic pixel size). Samples were exposed for typically $50 \mathrm{~s}$ with a cycle time of $1 \mathrm{~min}$.

\subsection{SAXS}

SAXS was performed at the synchrotron beamline A2 at HASYLAB, Hamburg. The wavelength was $0.15 \mathrm{~nm}$. The sample-detector distance ranged between 2 and $3 \mathrm{~m}$. Collection of the scattering patterns was identical to the procedure adopted for the USAXS experiment.

\subsection{Pre-evaluation}

The measured machine background weighted by the absorption factor was subtracted from the raw scattering patterns. Invalid pixels (e.g. those behind the beam stop) were discarded. The pattern was aligned by moving its physical center to the center of the image map, rotated so that the fiber axis became the vertical axis, and harmonized. The lastmentioned step comprises the filling of missing pixels by utilization of the four-quadrant symmetry of the fiber pattern. Finally, the scattering intensity was normalized to constant sample thickness by means of the absorption factor as determined from the readings of ionization chamber monitors (Stribeck, 2007).

\subsection{Beam profile measurement}

In order to determine the actual primary beam profile, a scintillation screen was provided with a ruler and placed in the position of the detector. The image of the beam spot was monitored by a TV camera, and a digitized video frame, $W_{\mathrm{V}}\left(x_{1}, x_{3}\right)$, was obtained. Here $x_{1}$ and $x_{3}$ are the horizontal and the vertical coordinates of the beam spot image expressed in absolute units of length. Fig. 2 shows the raw snapshot of a rather fine primary beam profile recorded at beamline A2 after a good adjustment. At its center $W_{\mathrm{V}}\left(x_{1}, x_{3}\right)$ is overexposed. Therefore, an analytical model of the beam profile was defined and fitted to the beam profile snapshot. Because the beam shaping is carried out by means of horizontal and vertical slits, the sought point spread function (PSF) is modeled by

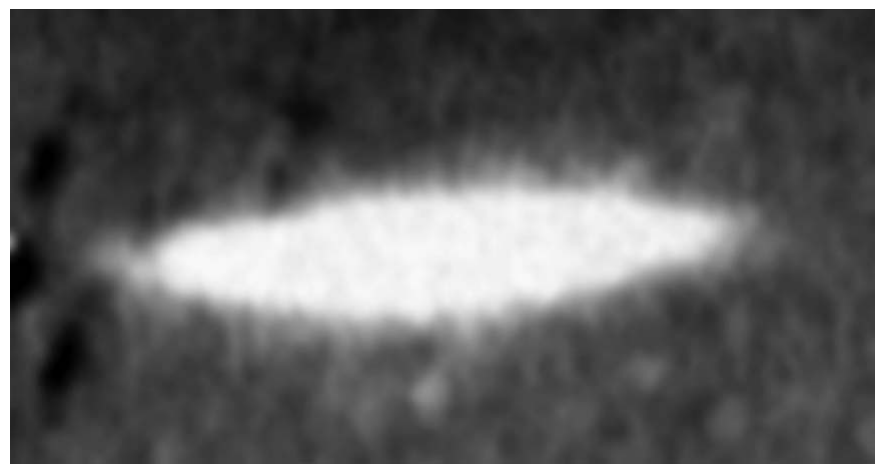

Figure 2

Digitized image of a primary beam profile at beamline A2, HASYLAB. The length of the bright spot is $3 \mathrm{~mm}$.

$$
W\left(x_{1}, x_{3}\right)=W_{1}\left(x_{1}\right) W_{3}\left(x_{3}\right),
$$

a product of coordinate functions. Moreover, from the calculation of the beamline optics it is known that each of these functions is closely approximated by the shape of a Gaussian.

After a transformation to the units of reciprocal space, $s=(2 / \lambda) \sin \theta$ with $\lambda$ the wavelength of radiation and $2 \theta$ the scattering angle, the PSF is

$$
W\left(s_{1}, s_{3}\right)=\frac{1}{2 \pi \sigma_{1} \sigma_{3}} \exp \left[-\frac{1}{2}\left(\frac{s_{1}}{\sigma_{1}}\right)^{2}\right] \exp \left[-\frac{1}{2}\left(\frac{s_{3}}{\sigma_{3}}\right)^{2}\right],
$$

with $s_{1}$ and $s_{3}$ the components of the scattering vector $\mathbf{s}$ in the equatorial and meridional directions, respectively.

For the profile presented in Fig. 2, we determine the standard deviations $\sigma_{1}=2.1 \times 10^{-3} \mathrm{~nm}^{-1}$ and $\sigma_{3}=5.9 \times$ $10^{-4} \mathrm{~nm}^{-1}$ in the horizontal and vertical directions, respectively. With respect to the pattern merging procedure described in $\S 3$, we are using this set of parameters as the starting value set.

\subsection{Smearing and desmearing}

The imperfect collimation quantified by the primary beam profile $W\left(s_{1}, s_{3}\right)$ causes the measured scattering pattern, $I_{\text {obs }}\left(s_{1}, s_{3}\right)$, to appear smeared (Alexander, 1979; Glatter \& Kratky, 1982; Baltá Calleja \& Vonk, 1989; Stribeck, 2007). Its relation to the sought-after ideal intensity, $I\left(s_{1}, s_{3}\right)$, is given by the correlation integral

$$
\begin{aligned}
I_{\mathrm{obs}}\left(s_{1}, s_{3}\right) & =\int_{-\infty}^{\infty} I\left(y_{1}, y_{3}\right) W\left(s_{1}+y_{1}, s_{3}+y_{3}\right) \mathrm{d} y_{1} \mathrm{~d} y_{3} \\
& :=I\left(s_{1}, s_{3}\right) \star W\left(s_{1}, s_{3}\right) .
\end{aligned}
$$

Correlation is equivalent to convolution if at least one of its partners is an even function, e.g. $W(\mathbf{s})=W(-\mathbf{s})$. In SAXS, inversion of equation (3) is, in general, carried out iteratively by variants of the method devised by van Cittert (Burger \& van Cittert, 1932; Ergun, 1968; Glatter, 1974). The variants differ in the way in which the correction function is smoothed - a function that is computed in each iteration step. In the common algorithm of Glatter (1974) the smoothing is performed by convolution with a triangle. Modern signal processing manuals (VNI, 2007) recommend the use of an adapted digital filter. As recommended, we utilized a tenthorder adapted digital filter with standard parameters from the library of PV-WAVE (VNI, 2007).

The principle of the iterative desmearing is based on the obvious fact that the correlation of the sought-after intensity, $I(\mathbf{s})$, with the primary beam profile, $W(\mathbf{s})$, is broadening the signal $I_{\text {obs }}(\mathbf{s})$. The basic idea is to smear the already smeared signal $I_{\text {obs }}(\mathbf{s})$ a second time, then to determine the resulting intensity variation at each point of the pattern, yielding a correction function; after the above-mentioned smoothing step, the variation is inverted in order to obtain an improved estimate of the sought function. Evidently, this principle can be iterated and convergence is found after $m$ iterations if the estimated intensity $I^{m}(\mathbf{s})$ smeared by $W(\mathbf{s})$ is equal to the measured intensity: 


$$
I^{m}(\mathbf{s}) \star W(\mathbf{s})=I_{\mathrm{obs}}(\mathbf{s}) .
$$

The result of the iterative method can, in general, be accepted because of the built-in check used in the convergence test, as long as the algorithm does not stop prematurely because of divergence. Premature divergence of an iterative algorithm is frequently caused by improper hole filling. In this case artifacts from outside the borders of the sensitive detector area creep into the image step by step and, finally, limit the success of the desmearing process.

\section{The pattern merging method}

Fig. 3 is a flowchart illustrating both the obvious straightforward pattern merging procedure (curved arrows) and a new complex pattern merging method proposed by us.

It has been necessary to develop the complex procedure because the simple method is unstable in practice, whereas the complex procedure has proven robust. Thus, it can be utilized for automatic processing of extensive series of scattering patterns from time-resolved experiments. Unfortunately, in the SAXS pattern the blind hole is large, and the data must be desmeared. This is only possible after filling the hole. In the complex procedure, the central hole in the SAXS pattern is filled using smeared USAXS data of the same sample in the same state, whereas the simple procedure is based on filling by data extrapolation.

Because desmearing is the inversion of an integration, the hole-filling procedure has to return good estimates. Even small errors concerning differentiability at the border of the sensitive region of the detector will turn into artifacts during desmearing. Another reason for seeking a suitable extrapolation method is that bad extrapolation causes early divergence of the iteration procedure and thus remnant artifacts, as explained in $\$ 2.6$.

In the following we present a new two-dimensional extrapolation method. It only appears suitable for filling the beamstop area of scattering patterns which do not require desmearing. Later in our data evaluation we use it for filling the hole in the center of the USAXS pattern.

Beyond that, a new method for the determination of an unknown primary beam profile (i.e. the PSF) of the SAXS pattern is presented.

\subsection{Improved blind-hole extrapolation}

Until recently we have used a standard method, based on radial basis functions (Buhmann, 2000; VNI, 2007), for twodimensional extrapolation. Figuratively, the procedure fills the blind region by some kind of stiffened 'rubber cloth'. For a direct analysis of the multidimensional chord distribution function (CDF; Stribeck, 2001) this practice is acceptable. Obviously, it is unsuitable if we are going to desmear a scattering pattern. Therefore, we have developed an advanced method which is less prone to produce desmearing artifacts. Figuratively, this procedure fills the hole by an 'igloo tent' supported by bent tent rods. Fig. 4 demonstrates both the

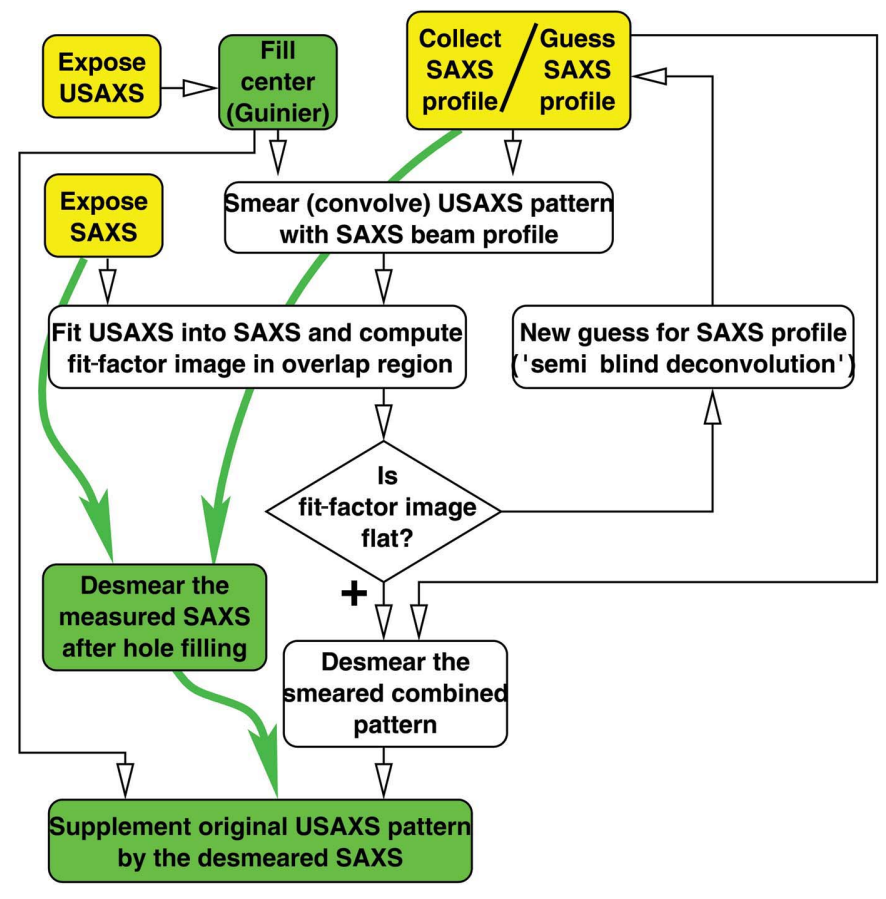

Figure 3

Flow chart of USAXS and SAXS pattern merging combined with collimation correction by desmearing. The straightforward method (curved arrows) suffers from artifacts originating from insufficient hole filling.

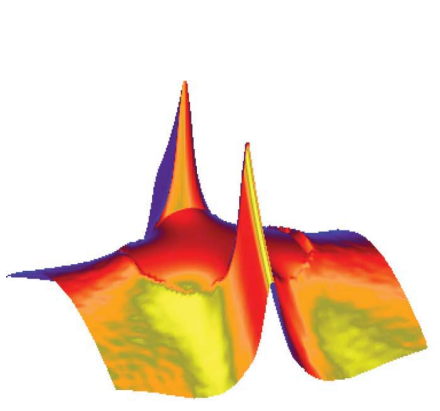

(a)

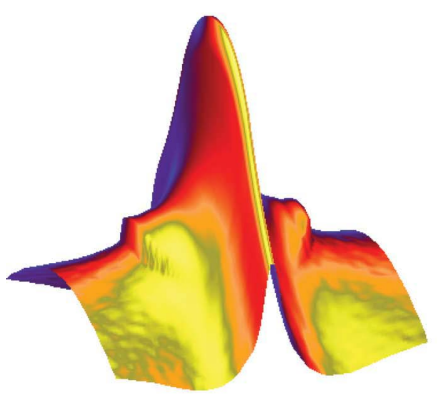

(c)

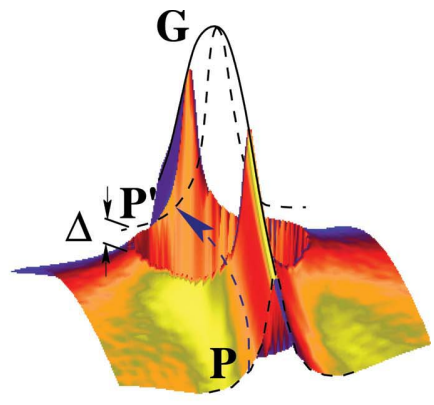

(b)

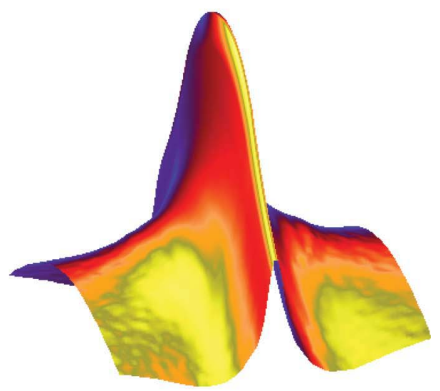

(d)
Figure 4

Filling of the central hole. (a) The common two-dimensional extrapolation by a 'rubber cloth' is only good for filling the hole on a high intensity level. (b) The central hole and the concept of filling by a 'tent rod' generated by Guinier extrapolation $(\mathrm{G})$, on which another tent $\operatorname{rod}\left(\mathrm{P}^{\prime}\right)$ is moving in a transverse direction. The shape of this rod is borrowed from valid data $(\mathrm{P})$. The remaining error $\Delta$ produces an artificial step. (c) The pattern before and $(d)$ the pattern after error correction by affine transformation of $\mathrm{P}^{\prime}$. 
previously used method for two-dimensional extrapolation (Fig. $4 a$ ) and the new extrapolation procedure.

The new extrapolation is carried out as follows. A fourthorder even polynomial is fitted to the meridional and to the equatorial sections of the pattern. The parabola that climbs highest [Fig. 4(b), labeled G] is retained and becomes the principal direction. The resulting curve resembles the beginning of the series expansion according to Guinier \& Fournet (1955). In the secondary direction, a complete profile $(\mathrm{P})$ is extracted from the measured data and put $\left(\mathrm{P}^{\prime}\right)$ on each point of the Guinier parabola. Now, parallel to the principal direction, a step of varying height $\Delta$ is observed (Fig. $4 b$ ). Finally, after affine deformation of each $\mathrm{P}^{\prime}$ (by multiplication with its local compensation factor), the filling smoothly approaches the measured data. The result is shown in Fig. 4(d).

We do not claim that such Guinier extrapolation has any physical basis in scattering theory when it is applied to the SAXS of polymers. Nevertheless, it appears more suitable for the approximation of the central scattering than the standard hole-filling method (Fig. 4a). Its intensity increases strongly towards the center, and concerning the derivative there is no discontinuity at the border.

\subsection{Hole-filling method compatible with desmearing}

A hole-filling method is compatible with desmearing if it does not introduce perturbing artifacts to the desmeared scattering pattern. This can easily be tested by means of, first, desmearing and, second, inspection of the central part of the desmeared SAXS pattern. Fig. 5 demonstrates the differences resulting from different hole-filling methods. The leftmost column (Figs. $5 a$ and $5 b$ ) shows the original smeared SAXS in the vicinity of the blind hole. Filled by different methods and desmeared, the results are displayed in the columns to the right.

The next column (Figs. $5 c$ and $5 d$ ) shows the result after filling by the radial-basis-function method. Considerable distortion is observed inside and outside the former blind spot. The distortion is manifested in strong 'over-desmearing', which even causes the apparent intensity to become negative.

Applying the new hole-filling procedure ( $c f$. Fig. 4 ) to the large SAXS hole, the remnant artifacts are less severe. They no longer cause meaningless intensity values (Figs. $5 e$ and $5 f$ ). Nevertheless, the area of the former hole is full of artificial reflections.

Unfortunately, a smooth appearance outside the hole area is not sufficient to guarantee satisfactory desmearing. In the next step of data evaluation, in which SAXS and USAXS patterns are merged, it sometimes transpires that there is a mismatch of the slopes in the overlap region. Thus, for automatic data processing, a more reliable hole-filling method is required.

Finally, if the USAXS pattern is smeared and then taken to fill the SAXS hole ( $c f$. Fig. $4 b)$, Figs. $5(g)$ and $5(h)$ show that inside the blind hole we still observe shallow artificial modulations. Thus, the affected region is replaced by the measured USAXS data. Outside the beam-stop area the artifacts are very weak. Consequently, the desmeared SAXS and the

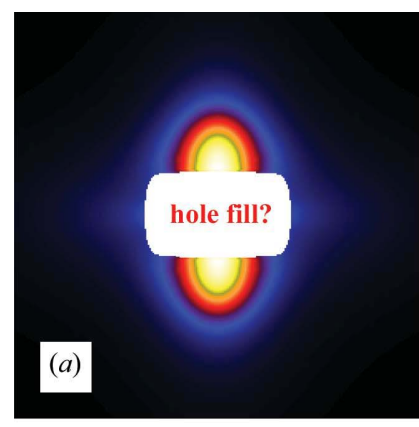

smeared

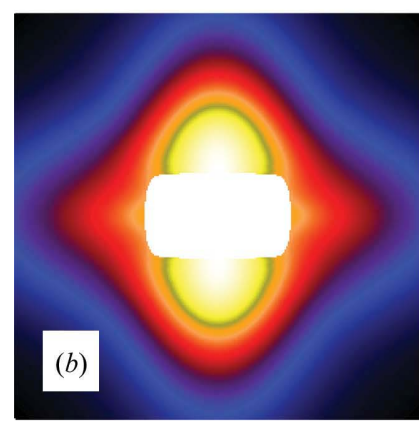

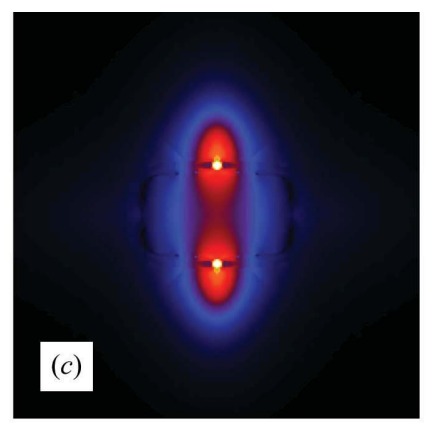

: rubber cloth

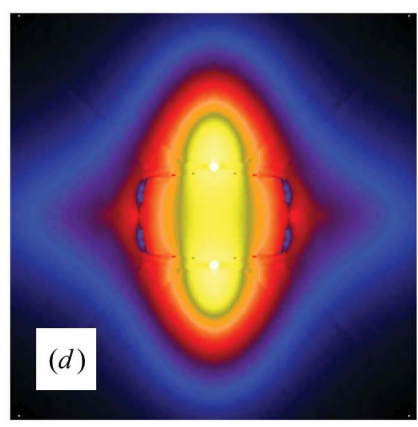

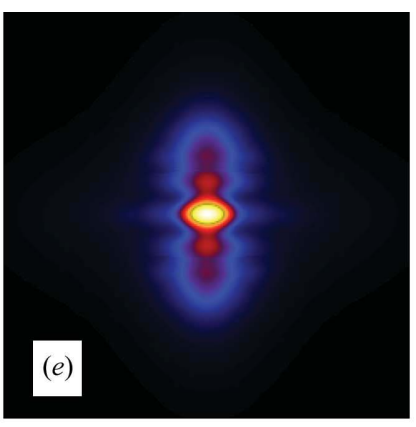

desmeared $\longrightarrow$ : igloo tent

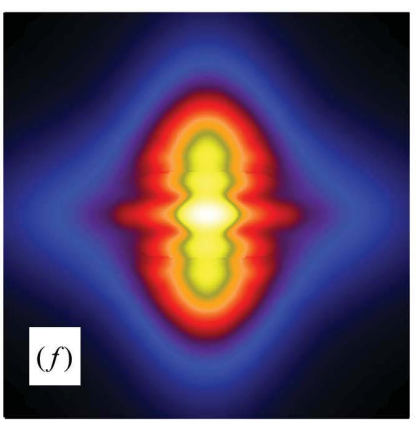

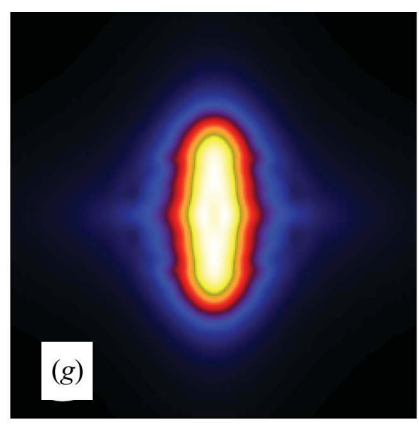

: true USAXS

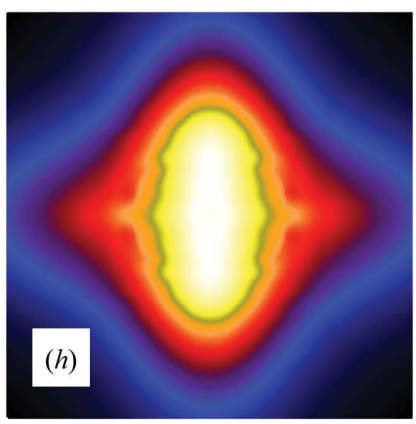

Figure 5

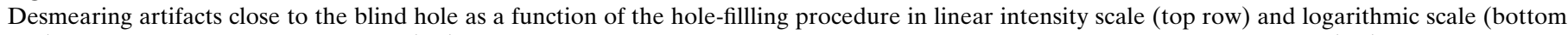

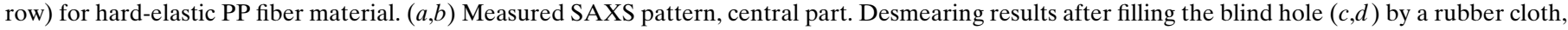
$(e, f)$ by the two-dimensional Guinier estimate and $(g, h)$ by the smeared USAXS of the same sample. 
USAXS can be matched and merged after simple multiplication by an almost constant factor.

Close to the outer edge of the detector area, cut-off artifacts are generated if no extrapolation is carried out. The corresponding intensity undulations can be suppressed by introduction of cyclic boundary conditions and extrapolation of missing data in the remaining corners of the pixel matrix. For this purpose the rubber-cloth extrapolation (Buhmann, 2000; VNI, 2007) works sufficiently, provided all data points have been eliminated that are affected by the penumbra close to the circumference of the vacuum tube.

\subsection{Filling by smeared USAXS and semi-blind deconvolution}

Fig. 6 demonstrates the filling of the central hole for a studied polypropylene nanofiber. This example is extreme, because the USAXS of the material is particularly distinct (Fig. 6a). Moreover, the adjustment of the beamline optics has been rather poor during the measurement of the SAXS (Fig. 6b). The scattering pattern of Fig. 6(c) was obtained by smearing of the measured USAXS pattern with the primary beam profile of the SAXS according to equation (3). It is readily fitted into the hole of the measured SAXS pattern. The result is shown in Fig. 6(d).

In order to merge the patterns, the average fit factor is computed from the region where both SAXS data and USAXS data are present.

Moreover, an individual fit factor for each pixel in the overlap zone can be computed. The result is a fill-factor matrix. The variation of this fill-factor matrix as a function of primary beam length and height is shown in Fig. 7. The fillfactor matrix at the center of the figure is the flattest, because there the parameters of the real PSF of the SAXS, $\sigma_{1}=3.1 \times$ $10^{-3} \mathrm{~nm}^{-1}$ and $\sigma_{3}=3 \times 10^{-3} \mathrm{~nm}^{-1}$, are used. Admittedly, even in this matrix shallow local maxima and minima are observed. Nevertheless, the amplitude between the highest peak and the deepest valley is smallest, and the peaks and the valleys are broader compared with the other fit factor matrices. Deviation from the optimum leads to an increasingly wavy fill-factor image. Thus, the flatness of the fill-factor matrix appears to be a suitable criterion for having chosen a primary beam profile of appropriate width and height. In this way a suitable SAXSPSF, $W(\mathbf{s})$, can be determined even if it has not been measured. After the determination, the SAXS pattern is desmeared using this PSF. We call this PSF search method a 'semi-blind' deconvolution, in contrast to the PSF search in the blind deconvolution which depends on the detection of overdesmearing.

In detail, as the beam is made longer and longer (Fig. 7, middle row), the fill-factor matrix compensates for this change by lifting the main peaks. On the other hand, as the primary beam height is increased (middle column), the overcompensation of the equatorial streak is first corrected and then lifted above the average fill factor. With an understanding of this mechanism, the direction of optimization can be determined after the first test with some starting values for the length and height of the primary beam.

\section{Results and discussion}

The described method is advantageously applied whenever both SAXS and USAXS exhibit discrete scattering ('doubleband scattering').

\subsection{Polypropylene fiber}

Clear double-band scattering is shown by a hard-elastic polypropylene fiber (Figs. $8 a$ and $8 b$ ). Fig. 8(a) displays only the central part of the recorded SAXS. The USAXS data (Fig. 8b) show that the blind spot in the SAXS pattern is so large that an essential fraction of the discrete scattering is still missing. Conversely, the USAXS provides the scattering inside the blind spot but not the higher orders of the main SAXS reflections.

If we take each part in its entirety and compare the results, both matches and differences are observed. For the purpose of demonstration the nanostructure computed from each singleband experiment is displayed in real space by means of the multidimensional CDF (Stribeck, 2001, 2007) (Figs. $8 c$ and $8 d)$.

Obviously, the long period and the average layer thickness [label (1) in the graphs] can be extracted from both patterns with little difference. Nevertheless, the advanced topological information is quite different. From the SAXS CDF, weak correlation among neighboring lamellae would be deduced [label (2)] which is not visible in the USAXS. On the other hand, only the USAXS CDF clearly shows the counterpart of the equatorial streak [label (3)].

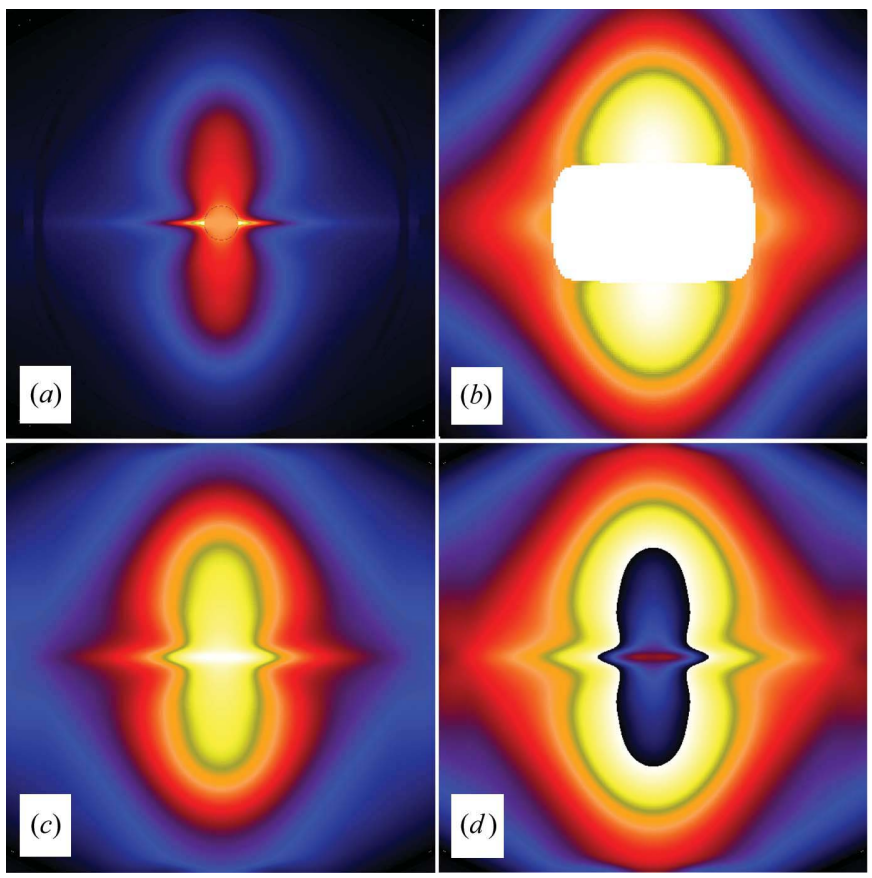

Figure 6

(a) USAXS pattern of a PP nanofiber measured at BW4. (b) The same sample measured at A2. (c) Pattern (a) smeared with the actual beam profile of beamline A2. (d) (b) filled with $(c)$. 
The result of merging USAXS and SAXS by means of the new method is shown in Fig. 9(a). Obviously, the novel twodimensional extrapolation method yields an acceptable intensity shape in the vicinity of zero scattering angle when applied to fill the small central hole in the USAXS. Moreover, in the transition region between USAXS and SAXS, even in the presented logarithmic scaling, neither a step nor a sudden change of slope is observed.

The CDF obtained from the merged pattern is shown in Fig. 9(b). In this CDF from the double-band (i.e. SAXS and USAXS) experiment, the nanostructural features of the previously discussed single-band CDFs are combined: both the weak correlation among the lamellae and the meridional double-ridge related to the needle-shaped domains that generate the equatorial streak in the USAXS are observed.

\subsection{Polypropylene nanofiber}

Finally, we present a material with even more pronounced discrete scattering in both angular ranges. Correspondingly, the filter effect of single-band experiments becomes even stronger. Similar effects can be expected with other ordered nanostructured polymeric materials that are presently the focus of research.

For such materials, even cursory inspection of the SAXS pattern clearly shows that the recorded data are incomplete,

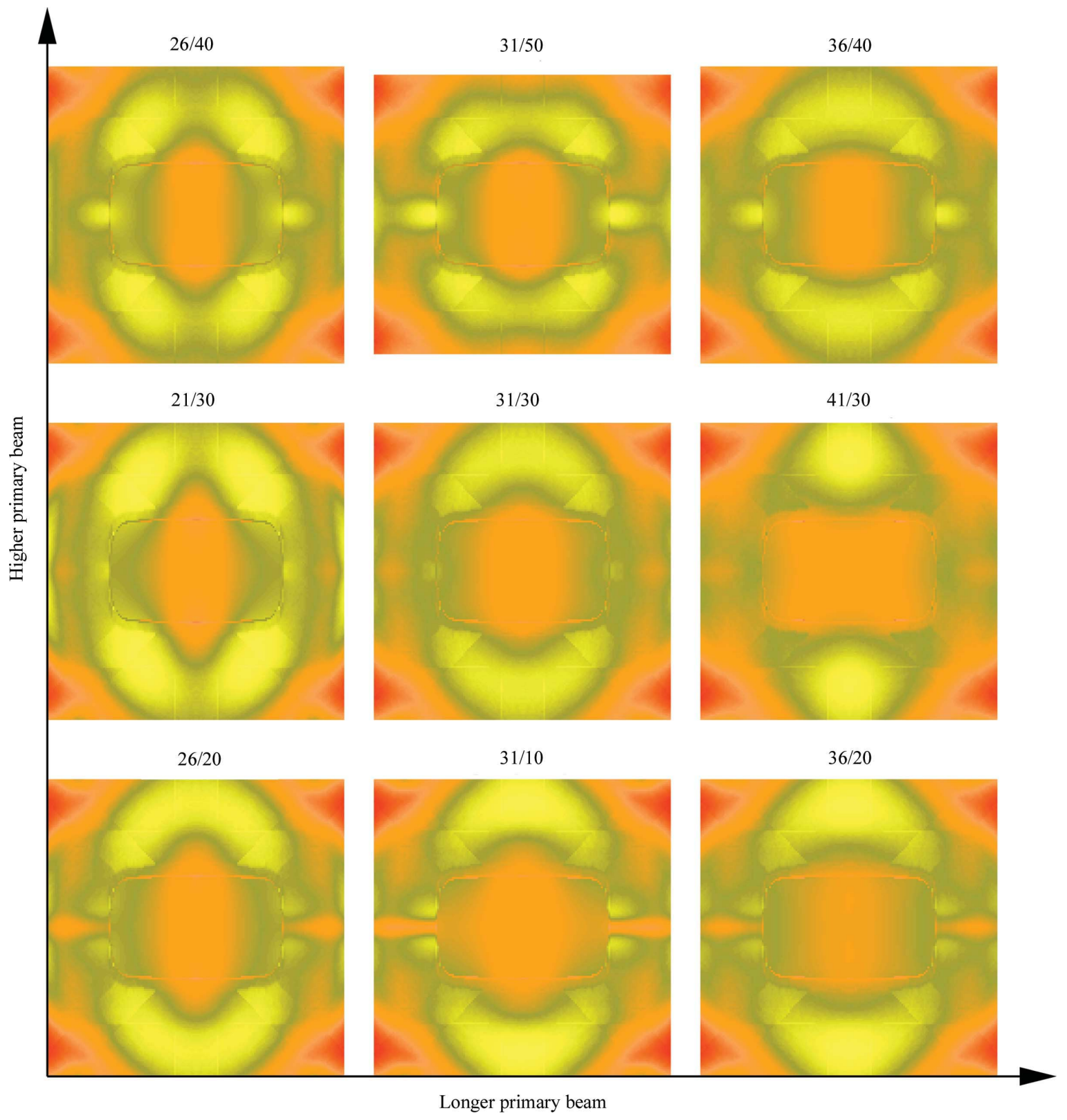

Figure 7

Demonstration of the semi-blind deconvolution approach in the desmearing procedure (Fig. 3). The size of the primary beam profile is chosen to be the one that flattens the image of fill factors best. Images of the fill factor are presented in pseudo-color as a function of width, $\sigma_{1}$, and height, $\sigma_{3}$, of the profile. The labels indicate the pairs $\sigma_{1} / \sigma_{3}$ in units of $10^{-4} \mathrm{~nm}^{-1}$ in reciprocal space. 
because not only part of the equatorial streak but also part of the long-period reflection are masked by the beam stop. On the other hand, if the USAXS is recorded first, the missing higher orders of the long-period reflection may be overlooked.

Without resorting to the scattering patterns, let us directly demonstrate the effect of band-pass filtering on the CDF

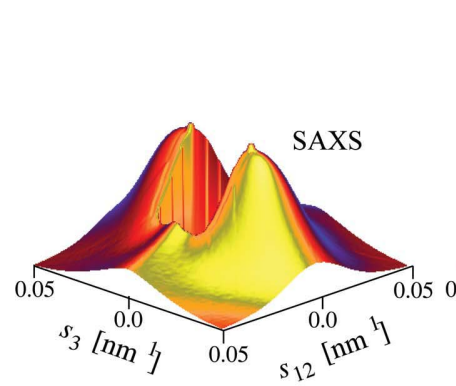

(a)

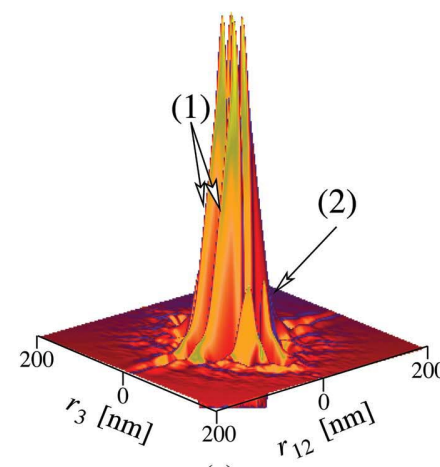

(c)

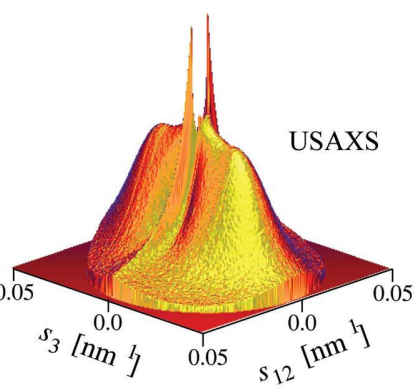

(b)

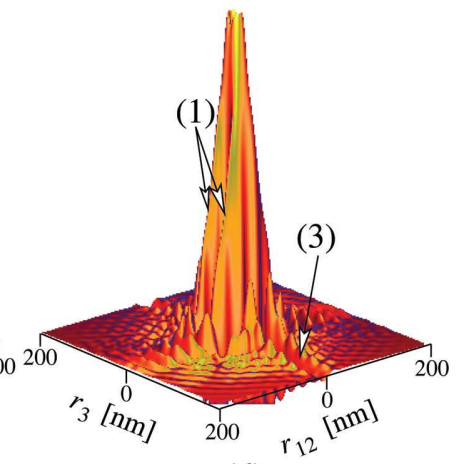

(d)
Figure 8

PP fiber. Comparison of the results of single-band SAXS and USAXS experiments. All data are shown on a logarithmic scale. $(a, b)$ Scattering data. $(c, d)$ The corresponding CDFs computed separately from each pattern. The sensitivity of each pattern to the features of the nanostructure is different: (1) The first pair of triangular peaks indicates the layer structure. (2) The second order indicates stacks of layers. (3) The meridional streak indicates needle-shaped domains extending in the fiber direction.

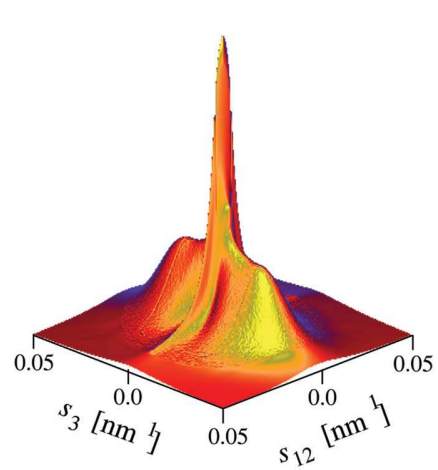

(a)

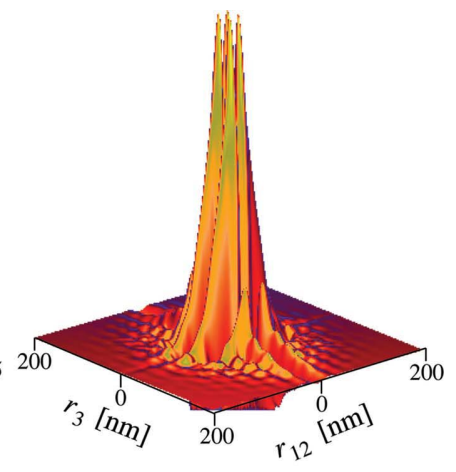

(b)
Figure 9

After pattern merging. (a) The inner part of the scattering merged from USAXS and SAXS. In the center the filling of the small USAXS hole by the novel extrapolation method is visible. The outward part of the pattern originates from the desmeared SAXS. (b) The CDF computed from the merged scattering pattern shows a combination of all the nanostructural features observed in Figs. 8(c) and $8(d)$ and a reduced level of artifacts.

nanostructure image. Fig. 10(a) exhibits the nanostructure of a pure lamella system. The layer thickness distribution appears to be rather broad. Only a shallow groove [Fig. 10(a), label (1)] indicates that the material might be made from stacks of finite lamellae with some transverse offset.

On the other hand, the USAXS CDF (Fig. 10b) shows a nanostructure that is dominated by the effect of needleshaped domains oriented in the fiber direction $\left(r_{3}\right)$. For the purpose of direct comparison we have chosen to display the same region of real space in all plots. On the basis of Fig. 10(b) we cannot discuss the details of the needle structure, but the peaks forming triangles in the equatorial direction $\left(r_{12}\right)$ are clearly visible and demonstrate that even the single-band USAXS detects some of the features of the lamella system.

Finally, the CDF based on the merged data (Fig. 10c) displays the complexity of the nanostructure of this material in high spatial resolution. Triangles (Stribeck, 2001) of narrow extension in the fiber direction characterize lamellae of rather uniform thickness [Fig. 10(c), $\left(1^{\prime}\right)$ ]. The bulky peaks in front of them [label $\left.\left(2^{\prime}\right)\right]$, which are split at the meridian, correspond to thicker intermediate layers with a broad thickness variation; the splitting results from individual transverse offsets (Stribeck et al., 2005; Keum et al., 2005) among the other layers, i.e. those of well defined thickness. Consequently, the crystalline layers, $\left(1^{\prime}\right)$, now appear distinguishable from the amorphous gaps, $\left(2^{\prime}\right)$, between them.

Moreover, the modulation of the triangular layer peaks [Fig. 10(c), (3)] indicates either that the lamellae are composed of blocks (Hugel et al., 1999) or that a cross-hatched structure (Olley \& Bassett, 1989) is present. Which explanation is more probable can be deduced from the structure

(a)

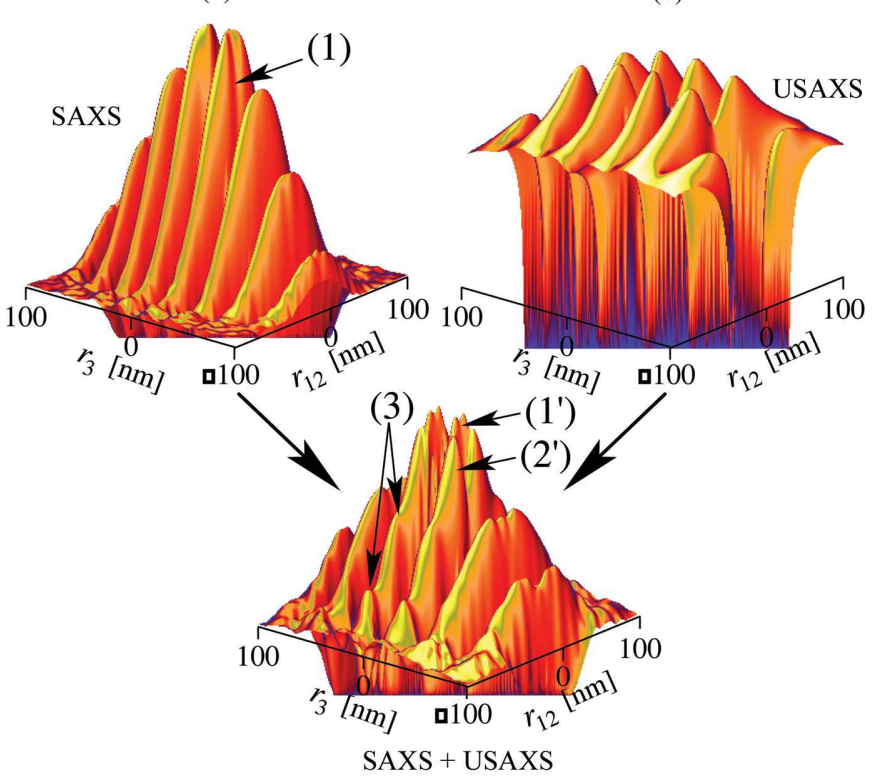

Figure 10

PP nanofiber material. Nanostructure information extracted from $(a)$ the SAXS pattern, $(b)$ the USAXS pattern and $(c)$ the merged scattering pattern. The real-space CDFs are trimmed to the same region. 
evolution during heat-treatment of the material, which has not yet been published.

The completeness of the double-band data would be guaranteed if we could verify that outside the limits of the combined angular band sensed in our experiments $(0.001<$ $|\mathbf{s}|<0.3 \mathrm{~nm}^{-1}$ ) there is no discrete scattering. In this case even an extension of the angular bandwidth would not further clarify the perception of the nanostructure.

\section{Conclusion and outlook}

The discrete modulation of the intensity measured in a scattering experiment is, in principle, the power spectral density of the information on the arrangement and shape of the domains in the material. We assume that this information is bandlimited. In the case where the bandwidth of the studied material is wider than the angular band of the instrument employed, alteration of the reconstructed nanostructure cannot be avoided. In this paper we have proposed a method that can be used to minimize the corresponding shift by means of tandem experiments, via desmearing and merging of the scattering patterns. The proposed method of iterative determination of the desmearing operator from comparison of smeared SAXS and ideal USAXS data (semi-blind deconvolution) provides accurately merged data covering a wide angular range.

In order to avoid the proposed complex procedure one might think of increasing the angular bandwidth of the instrument by employing both a microbeam and a large-area high-resolution detector (Riekel, 2000). In such a setup, USAXS and SAXS would be recorded simultaneously. Nevertheless, a new problem arises with such a setup, from the steep fall-off of the scattering intensity and the necessity to collect low-noise data over the complete angular range. In the case of static measurements and if a detector with high (or cyclic) dynamics were at hand, one could simply wait until even at high angles enough photons had been collected. On the other hand, longer exposure is no solution if structure evolution is to be studied in situ. In this area of research the available devices even for the singe-band setup are still slow. Thus, tandem experiments carried out at dedicated synchrotron beamlines appear to be a serious option for the investigation of nanostructure evolution of complex polymer materials.

Another pathway may become viable as detector technology and data treatment advance. With the novel PILATUS detector technology (Broennimann et al., 2006), large-area detectors with a short readout time can be realized, and by application of three-dimensional adapted smoothing techniques it may become possible to produce low-noise sequences of double-band scattering data with high time resolution in a single experiment - a microbeam setup with a large, fast and effective high-resolution detector. The addressed three-dimensional smoothing procedure would include the elapsed time, $t$, as the third dimension, considering the measured intensity $I\left(s_{12}, s_{3}, t\right)$ as a function of three variables. The method could be adapted by increasing the number of considered pixels for smoothing with increasing distance $\left(s_{12}^{2}+s_{3}^{2}\right)^{1 / 2}$ from the center of the pattern.

We thank the Hamburg Synchrotron Radiation Laboratory (HASYLAB) for beam time granted in the framework of project II-01-041. We are in particular obliged to the beamline scientists S. S. Funari and A. Timmann for their support. We gratefully acknowledge funding by the Deutsche Forschungsgemeinschaft, project STR 501/4-2.

\section{References}

Alexander, L. E. (1979). X-ray Diffraction Methods in Polymer Science. New York: Wiley.

Baltá Calleja, F. J. \& Vonk, C. G. (1989). X-ray Scattering of Synthetic Polymers. Amsterdam: Elsevier.

Broennimann, Ch., Eikenberry, E. F., Henrich, B., Horisberger, R., Huelsen, G., Pohl, E., Schmitt, B., Schulze-Briese, C., Suzuki, M., Tomizaki, T., Toyokawa, H. \& Wagner, A. (2006). J. Synchrotron Rad. 13, 120-130.

Buhmann, M. D. (2000). Acta Numerica, 9, 1-38.

Burger, H. C. \& van Cittert, P. H. (1932). Z. Phys. 79, 722.

Deutsch, M. \& Luban, M. (1978). J. Appl. Cryst. 11, 98-101.

Ergun, S. (1968). J. Appl. Cryst. 1, 19-23.

Glatter, O. (1974). J. Appl. Cryst. 7, 147-153.

Glatter, O. \& Kratky, O. (1982). Editors. Small Angle X-ray Scattering. London: Academic Press.

Guinier, A. \& Fournet, G. (1955). Small-Angle Scattering of X-rays. London: Chapman and Hall.

Hugel, T., Strobl, G. \& Thomann, R. (1999). Acta Polym. 50, 214-217.

Keum, J. K., Burger, C., Hsiao, B. S., Somani, R., Yang, L., Chu, B., Kolb, R., Chen, H. \& Lue, C.-T. (2005). Prog. Colloid Polym. Sci. 130, 113-125.

Kratky, O., Porod, G. \& Kahovec, L. (1951). Z. Elektrochem. 55, $53-$ 59.

Olley, R. H. \& Bassett, D. C. (1989). Polymer, 30, 399-409.

Porod, G. (1967). Electromagnetic Scattering. Proceedings of ICES 2, Amherst, MA, June 1965, edited by R. R. Rowell \& R. S. Stein, pp. 319-337. New York: Gordon and Breach.

Riekel, C. (2000). Rep. Prog. Phys. 63, 233-262.

Ruland, W. (1964). Acta Cryst. 17, 138-142.

Ruland, W. (1977). Colloid Polym. Sci. 255, 417-427.

Soliman, M., Jungnickel, B.-J. \& Meister, E. (1998). Acta Cryst. A54, 675-681.

Stribeck, N. (2001). J. Appl. Cryst. 34, 496-503.

Stribeck, N. (2007). X-ray Scattering of Soft Matter. Heidelberg, New York: Springer.

Stribeck, N., Bösecke, P., Bayer, R. \& Almendarez Camarillo, A. (2005). Prog. Collect. Polym. Sci. 130, 127-139.

Syneček, V. (1960). Acta Cryst. 13, 378-380.

VNI (2007). $P V$-WAVE Manuals. Version 7.5. Visual Numerics Inc., Boulder, Colorado, USA. 